\title{
Measuring Risk of Portfolio : GARCH-Copula Model
}

\author{
Samia Ben Messaoud \\ Faculty of Economics and Management of tunis, Tunis, Tunisia \\ Chaker Aloui \\ College of Business Admnistration, King Saud University, Riyadh, KSA
}

\begin{abstract}
In this paper, we use the copulas functions in financial application, namely to examine the assumption of asymmetric dependence and to calculate some measures of risk. The first step consists of deducing filtered residuals for each return series by an asymmetric Glosten-Jagannathan-Runkle Generalized Autoregressive conditional Hetero skedasticity (GJR-GARCH) model. For the second step, we use an estimation of a Generalized Pareto Distribution for the upper and lower tails to determine the empirical semiparametric marginal Cumulative Distribution Function. In our approach, we propose to use a portfolio consisting of increments from five countries. The GJR-GARCH copula is then applied to the data and used to reduce correlation between the simulated residuals of each series. The marginal distributions of filtered residuals are fitted with a semi-parametric Cumulative Distribution Function using the copulas' functions and Generalized Pareto Distribution for tails. For each series, we compute Value-at-Risk and Conditional Value-at-Risk.
\end{abstract}

\footnotetext{
* Corresponding Author: Samia Ben Messaoud; Department of Economics, International Finance Grouup-Tunisia, Faculty of Management and Economics Sciences of Tunis, E1 Manar university, E1 Manar, PB.P 248. C.P.2092, Tunis, Tunisia; Tel: +216 20874894, Fax: +216 71870277, E-mail: samia.benmessaoud@yahoo.fr.

Co-Author: Chaker Aloui; Department of Economics, College of Business Administration, King Saud University, Kingdom of Saudi Arabia; Tel: +966 4693887, Fax: +966 4693443, E-mail: chakeraloui2@yahoo.fr.
} 
JEL Classification: E32, F15, F42

Keywords: GJR-GARCH, Copula Model, Portfolio Risk, Value at Risk, Conditional Value at Risk

\section{Introduction}

Market risk managers have to comprehend the stochastic confidence in the markets' risk-sensitivity in their portfolio to calculate risk measures as value-at-risk. In the recent years, many essays developed an approach to measure the risk of random events via Jorion (1996) and Dowd (1998).

Value-at-Risk is a statistic model which is described to declare the risk of an exposure by a single number, that is, Value-at-Risk model estimates the potential loss for a financial instrument portfolio over a given time horizon and confidence level. It became the standard and simple measurement in risk management widely used in financial sectors. However, it has many limitations in quantifying the risks. In 2001, Dembo and Freeman showed that Value-at-Risk models do not present a sufficient distinction between good and bad risks. It is not a coherent risk measure because it does not satisfy one of axiomatic condition; namely, sub-additivity. Also, it only describes a limit of the losses but do not define the potential loss when the limit is exceeded. Just like in Long Term Capital Management (LTCM) case, the collateralized debt obligations of Barings Bank, Enron, Bear Sterns, or Lehman Brothers were a resounding disaster in which correlations and risk in general were not faithfully measured.

Extreme values is important to contribute in this area by using the heterosckedasticity with some mathematical purpose to adjust so that it is consistent with reality.

As a solution, there are several volatility models from GARCH family such as asymmetric GARCH Glosten, Jagannathan, and Runkle (GJR 1993), exponential GARCH (EGARCH) (Nelson 1991), Fuzzy-GARCH, Markov-Switching GARCH, and other approaches to model the heteroskedasticity.

Also, we use the copulas in the multivariate case for computing the stochastic dependence between the variables. They present the way that the marginal distributions have to be coupled to yield the joint distribution function.

Our contribution in this paper is first the use of a copula function followed by the 
use of GARCH copulas, in particular, a GJR-GARCH to examine the asymmetric dependence between structure and measure of complex non-linear relationships among financial asset returns.

The present paper is organized as follows. Section II presents a short review of the relevant literature describing the proposed approach. The copulas employed in this paper are presented in Section III. Section IV contains the data processing and the empirical results. Finally, Section V concludes.

\section{Review for Copula}

In this section, we first present the fundamental relations, properties, and families of the copula and then review the application of the copula.

\section{A. Copula definition}

The copula is essentially a function that links univariate marginal distributions to the joint multivariate distribution function, which is simply a convenient way to describe joint distributions of two or more random variables. The theory of copula is a highly powerful tool for modeling joint distributions because it does not require the assumption of joint normality and allows us to decompose any $n$-dimensional joint distribution into its $n$ marginal distributions. It is able to extract the dependence structure from the joint probability distribution function and, simultaneously, to isolate such dependence structure from the univariate marginal distributions. Conversely, a copula produces a multivariate joint distribution, combining marginal distributions and dependence between the variables. The copula has been broadly used in the statistical literatures. The books of Joe (1997), Nelsen (1999), and Trivedi and Zimmer (2007) presented a good introduction to the copula theory. Although the copula has been recently used in the financial area, there are already several applications (Bouyè et al. 2000, Embrechts, McNeil, and Straumann 2002, and Embrechts, Lindskog, and McNeil 2001).

For simplicity purposes, we define only the bivariate case: A two-dimensional copula function is a function $C$, whose domains are $[0,1]^{2}$ and whose range is $[0,1]$. We also 
consider the following properties:

$$
\begin{aligned}
& C\left(x_{1}, 1\right)=C\left(1, x_{2}\right)=1 \\
& C(x)=0 \text { for all } x \in[0,1]^{2} \text { when at least one element of } x \text { is } 0 \\
& \qquad C\left(x_{1}, 1\right)=C\left(1, x_{2}\right)=1 \text { for all }\left(x_{1}, x_{2}\right) \in[0,1]^{2} \\
& \text { for all }\left(a_{1}, a_{2}\right),\left(b_{1}, b_{2}\right)=[0,1]^{2} \text { with } a_{1} \leq b_{1} \text { and } a_{2} \leq b_{2}
\end{aligned}
$$

We have:

$$
V_{C}([a, b])=C\left(a_{2}, b_{2}\right)-C\left(a_{1}, b_{2}\right)-C\left(a_{2}, b_{1}\right)+C\left(a_{1}, b_{1}\right) \geq 0
$$

Also, the copula $C$ can define as a function for the random vector $\mathrm{X}=\left(X_{1}, X_{2}\right)^{t}$, if it is the joint distribution function of the random vector $\mathrm{U}=\left(U_{1}, U_{2}\right)^{t}$ where $U_{i}=F_{i}(X)$, and $F i$ are the marginal distribution functions of $X_{i}, i=1,2$.

Such that:

$$
F\left(x_{1}, x_{2}\right)=C\left(F_{1}\left(x_{1}\right), F_{2}\left(x_{2}\right)\right)
$$

Where $\mathrm{F}$ is the joint distribution function of $\left(X_{1}, X_{2}\right)$. If $F_{1}$ and $F_{2}$ are continuous then the copula $C$ is unique. Thus, we can interpret a copula as a function which links the marginal distributions of a random vector to its joint distribution.

Now, we present five types of dependence structures to estimate the joint distribution; namely, Gaussian Copula, student Copula, Clayton Copula, Frank Copula, and Gumbell copula.

\section{B. Copula families}

The Gaussian copula is the copula of the multivariate normal distribution which is defined by the following equation: 


$$
C_{\rho}\left(u_{1 t}, u_{2 t}, \ldots ; u_{n t}\right)=\Phi_{p}\left(\phi^{-1}\left(u_{1 t}\right), \phi^{-1}\left(u_{2 t}\right), \ldots, \phi^{-1}\left(u_{n t}\right)\right)
$$

$\phi$ : function of the normal distribution $N(0,1)$

$\phi^{-1}$ : the inverse function of the normal cumulative distribution

$\Phi_{\mathrm{p}}$ : the distribution the centered Gaussian vector $(X, Y)$ of the covariance matrix

$$
\begin{gathered}
\left(\begin{array}{ll}
1 & \rho \\
\rho & 1
\end{array}\right) \\
\Phi_{p}=\int_{-\infty}^{x} \int_{-\infty}^{y} \frac{1}{2 \pi \sqrt{\left(1-\rho^{2}\right)}} \exp \left[-\frac{\left(s^{2}+t^{2}-2 \rho s t\right)}{2\left(1-\rho^{2}\right)}\right] d s d t
\end{gathered}
$$

To capture the fat tail property, we introduce the multivariate student's $t$ Copula which shows more observations in the tails than the Gaussian. The student's $t$-copula is defined as,

$$
\begin{gathered}
C\left(u_{1 t}, u_{2 t}, \ldots, u_{n t}\right)=T_{\rho, k}\left(T_{\rho}^{-1}\left(u_{1 t}\right), T_{\rho}^{-1}\left(u_{2 t}\right), \ldots . ., T_{\rho}^{-1}\left(u_{n t}\right)\right) \\
t_{\rho, v}(x, y)=\int_{-\infty}^{x} \int_{-\infty}^{y} \frac{1}{2 \pi \sqrt{1-\rho^{2}}}\left(1+\frac{s^{2}+t^{2}-2 \rho s t}{v\left(1-\rho^{2}\right)}\right)^{-\frac{v+2}{2}} d s d t
\end{gathered}
$$

$t_{\rho, v}$ is a standard multivariate $t$ distribution, while the correlation $\rho$, and $v$ is the degrees of freedom.

It is very close to a Gaussian with strong correlations for movements with similar signs. The relationship that applies for Kendall's tau for a Gaussian copula also applies for student copula. The $t$ copula is a student copula which joins the marginal $t$ distributions with same degrees of freedom to the bivariate $t$ distribution. The $t$ student copula generalizes the bivariate $t$ distribution because we can adopt any marginal distribution. The Joe-Clayton copula function is given by the following Cumulative Distribution Function (CDF): 


$$
C_{\theta}\left(u_{1}, u_{2}, \ldots . ., u_{n}\right)=\left(u_{1}^{-\theta}+u_{2}^{-\theta}+\ldots+u_{n}^{-\theta}-n+1\right)^{-\frac{1}{\theta}}
$$

$$
\text { Its generator is, } \phi(x)=\frac{u^{-\theta}-1}{\theta}
$$

At this level, copulas have been proven to be a good technique to represent dependence between the variables' contributions to the two models of the system: the simple and the complex. In reliability system models, they alternate between whether common reasons are unknown or destined to be modelled.

The Frank copula is described by the following equation:

$$
C_{\text {Frank }}\left(u_{1}, u_{2} ; \lambda\right)=\frac{-1}{\lambda} \log \left(\frac{\lambda\left(1-e^{-\lambda}\right)-\left(1-e^{-\lambda u_{1}}\right)\left(1-e^{-\lambda u_{1}}\right)}{1-e^{-\lambda}}\right)
$$

$$
\text { Here, } \lambda \in(-\infty, 0) \cup(0,+\infty)
$$

The Gumbel copula was introduced in Hougaard (1986). It is also known as the Gumbel-Hougaard family. The CDFs are defined by the following:

$$
\begin{gathered}
C_{\text {Gumbel }}\left(u_{1}, u_{2} ; \delta\right)=\exp \left(-\left(\left(-\log u_{1}\right)^{\delta}+\left(-\log u_{2}\right)^{\delta}\right)^{\frac{1}{\delta}}\right) \\
C_{\text {Rotated-Gumbel }}\left(u_{1}, u_{2} ; \delta\right)=u_{1}+u_{2}-1+C_{\text {Gumbel }}\left(1-u_{1}, 1-u_{2} ; \delta\right) \\
\text { where } \delta \in[0, \infty)
\end{gathered}
$$

$\delta$ is the parameter which determines the correlation of random variables. $C\left(u_{1}, u_{2}, \ldots\right.$, $u, \delta$ ) is completely correlated. 
The recent papers apply the copula concept. Breymann et al. (2003) has considered the Gaussian, Student, Clayton, and Gumbel copulas. Among the various copulas, they judge that the student copula performs the best. Chen et al. (2004) consider 30 daily US stock returns and 20 daily exchange rate returns with the US Dollar as a base, and multivariate versions of the Gaussian and $t$-copula. They point out that the Gaussian copula is always rejected. The student copula assumes to be competent for analyzing the multivariate stochastic dependence of stock returns. Patton (2006) has studied the conditional and unconditional versions of the Gaussian copula and the symmetrized JoeClayton copula for defining the stochastic dependence between them.

Malevergne and Sornette (2003) finds that the bivariate student copula can be agreed upon for exchange rates and stocks if it has sufficient degree of freedom. Ane and Kharoubi (2003) examines unconditional versions of the Gaussian copula to evaluate the stochastic dependence between pairs of five stock indices. They achieve the Johnson copula, which exhibits lower tail dependence and upper tail independence. Van den Goorbergh (2004) examines that in general, a conditional student copula seems to be most suitable for defining the pair-wise dependencies. Jondeau and Rockinger (2006) finds that a conditional student copula performs better than a conditional Gaussian copula. Hotta and Palaro (2006) uses conditional copula to estimate Value-at-Risk for a bivariate indices portfolio. Rodriguez (2007) estimates the stochastic dependence between daily returns. He examines evidence for changing dependencies during the crisis. The Gaussian and the student copula exhibit features that violate the observed complex dependence structure which is characterized by asymmetry and upper tail dependence. Studies that analyze the default risk dependencies include those by Das et al. (2007) and Aboura and Wagner (2008). They show that observable macroeconomic variables and latent common variables play a major role in explaining correlated defaults in systematic credit risk.

\section{Copula applications}

Applications which used copula to estimate Value at Risk (VaR) go back the last ten years. According to Huang et al. (2009), copulas are methods that describe the dependence structure of a random multi-dimensional variable and they have become one of the most important tools to manage new risk factors in finance, like VaR which is probably the largest risk measure used by financial institutions. The VaR measures 
the potential loss in value of a risky asset or portfolio over a defined period for a given confidence interval.

The copula used by the previous studies does not include conditionality so they input no time-varying function. It is used with a short fall expected during the estimation of flat tails. Juri and Wuthrichts (2002) combines copula with Extreme Value Theory (EVT), while Mendes and Souza (2004) focused on crisis scenarios to calculate VaR quantity. Embrechts et al. (2005) used copula to estimate VaR in the worst case scenarios.

There are also several methods to determine the VaR. For instance, Cherubini and Luciano (2001) estimated the VaR using the Archimedean copula family and the historical empirical distribution in the estimation of marginal distributions; Rockinger and Jondeau (2001) used the Plackett copula with GARCH process with innovations modeled by the $t$ Student asymmetrical generalized distribution of Hansen (1994), proposing a new measure of conditional dependence; Georges (2001) used the normal copula to model the options time of exercise and for derivative pricing; Meneguzzo and Vecchiato (2002) used copula for modeling the Risk of Credit Derivatives (RCD); Fortin and Kuzmics (2002) employed convex linear combinations of copula for estimating the VaR of a portfolio composed by the FSTE and DAX stock indices; and Embrechts, McNeil, and Straumann (2002) and Embrechts, Hoing and Juri (2003) takes copula to model extreme value and risk limits. These recently published papers show a wide range of copula applications in finance.

The recent extension of the unconditional copula theory to the conditional case has been done by Patton (2003a) to model time-varying conditional dependence. Time variation in the first and second conditional moments is widely discussed in the statistical literature, so allowing the temporal variation in the conditional dependence in time series seems to be natural. Wang et al. (2010) applies a GARCH model to estimate exchange portfolio risk. Multivariate copulas, including Guassian and Clayton copula have been applied to describe portfolio risk structure and extend a bivariate analysis of an active dimensional allocation problem.

In this study, we use a GJR version of GARCH based on a student's $t$-copula to estimate VaR. This paper proposes an application of the copula-GARCH to estimate the VaR of a portfolio, which is our contribution. Compared to traditional methods, the results capture the copula model of VaR very well. Second, we combine the GARCH modeling approach and some multivariate extreme value copula functions. 


\section{Estimation Models}

\section{A. Copula parameter estimates}

The copula theory is a fundamental tool in modeling multivariate distributions. It allows the definition of the joint distribution through the marginal distributions and the dependence between the variables.

In application domains, conditional copula was constructed based on the Sklar's (1959) dependency theory. We use the Sklar's theorem which is a key result in the theory of copula.

The theorem of Sklar is described as follows: let $F$ be a complete bivariate joint distribution function having univariate marginal distributions $F_{1}$ and $F_{2}$. The copula $C$ related to $F$ is written as:

$$
\begin{aligned}
& C\left(u_{1}, \ldots \ldots \ldots, u_{d}\right)=C\left(F_{1}\left(x_{1}\right), F_{2}\left(x_{2}\right), \ldots \ldots \ldots . ., F_{d}\left(x_{d}\right)\right) \\
& =F\left(F_{1}^{-1}\left(x_{1}\right), F_{2}^{-1}\left(x_{2}\right), \ldots \ldots \ldots, F_{d}^{-1}\left(x_{d}\right)\right) \\
& =F\left(x_{1}, x_{2}, \ldots \ldots \ldots, x_{d}\right)
\end{aligned}
$$

With this theorem, we can conclude that for continuous multivariate distribution, the univariate marginal distribution and the multivariate dependence structure can be separated. This copula is unique if the margins are continuous. The proof can be found in Nelsen (1999).

It is the converse of the Sklar's theorem that is most interesting for modeling multivariate distributions in finance. It implies that we may link any group of $n$ univariate distributions, of any type of families, with any copula and we will have defined a valid multivariate distribution. The usefulness of this result stems from the fact that while in economics and statistics literature, we have a large set of flexible parametric univariate distributions available, the set of parametric multivariate distributions available is much smaller.

According to Patton (2003b), decomposing the multivariate distribution into the marginal distributions and the copula allows for the construction of better models of the individual variables than would be possible if we constrained ourselves to look only at existing multivariate distributions. 
Patton (2003a) made further extension and proved the validity of the Sklar's theorem for the conditional case. In this extension, the conditioning variable, $W$, must be the same for the marginal distributions and the copula.

For the definition of copula for the general case $(n>2)$ and for more details, look at Nelsen (1999) and Bouyé et al. (2000).

Density $f$ of a bivariate distribution might be written also as a function of density $C$ of the relevant copula and densities of $F_{1}$ and $F_{2}$.

$$
f\left(x_{1}, x_{2}\right)=C\left(F_{1}\left(x_{1}\right), F_{2}\left(x_{2}\right)\right)
$$

\section{B. Generalized pareto distribution}

Once the marginal distributions of filtered residuals were fitted using a Generalized Pareto Distribution (GPD) approach for tails distribution and a Gaussian kernel for the interior of distribution, the next stage was to estimate the parameters of dependence structure for the analyzed portfolio. While isolating the effects of marginal distribution, the dependence existent among the four currencies was estimated. Capturing the interaction among the portfolio assets by isolating the individual behavior of each asset is, in fact, the role of Copula.

We focus on the distribution of losses beyond a given threshold, $\mu$ :

$$
F_{\mu}(y)=P\{X-\mu \leq y / X \succ \mu\}
$$

For distributions checking extreme value theorem and for a threshold $u$ that is large enough, there is $x, b$ such that $F_{\mu}$ converges towards a Generalized Pareto Distribution.

$$
\begin{gathered}
F_{\mu}(y)=G_{\xi, \beta}(y) \\
G_{\xi, \beta}(x)=\left\{\begin{array}{l}
1-\left(1+\frac{\xi x}{\beta}\right)^{-\frac{1}{\xi}} \xi \neq 0 \\
1-\exp \left(-\frac{x}{\beta}\right) \xi=0
\end{array}\right.
\end{gathered}
$$


If $n$ is the total number of the sample and $N_{u}$ the number of values exceeding $u$, then:

$$
\begin{gathered}
\widehat{F}(x)=1-\frac{N_{u}}{N}\left(1+\xi \frac{x-\mu}{\widehat{\beta}}\right)^{-\frac{1}{\xi}} \\
\overline{V A R_{q}}=u+\frac{\widehat{\beta}}{\hat{\xi}}\left(\left(\frac{n}{N_{u}}(1-q)^{-\bar{\xi}}-1\right)\right)
\end{gathered}
$$

We may also estimate loss forecast in case of exceeding $\mathrm{VaR}$.

$$
\begin{gathered}
E S_{q}=V A R_{q}+E\left[X-\operatorname{VaR}_{q} / X \succ V a R_{q}\right] \\
\frac{E S_{q}}{V a R_{q}}=\frac{1}{1-\xi}+\frac{\beta-\xi u}{(1-\xi) V a R_{q}}
\end{gathered}
$$

\section{GJR-GARCH model}

Bollerslev (1986) developed GARCH as a generalization of Engle's (1982) original ARCH volatility modeling technique. He designed it to offer a more parsimonious model (using fewer parameters) that lessens the computational burden. Nelson (1991) proposed an Exponential GARCH (EGARCH) model, based on a logarithmic expression of the conditional variability in the variable under analysis. Later, a number of modifications were derived from this method. One of them is the GJR-GARCH model of Glosten, Jagannathan, and Runkle (1993) which can accommodate the asymmetry in the response of the variance to a shock.

Unlike the classical GARCH model, the GJR model contains an asymmetric effort. Here, asymmetry is captured by the term multiplying $\phi$. When $\phi$ is positive, it means that negative shocks introduce more volatility than positive shocks of the same size in the subsequent period. The estimation of the parameters above is also introduced in the following section. The conditional marginal distribution of $X_{t+1}$ is almost the same as the 
GARCH model, which is defined as the following.

Consider a GARCH model under the innovation normality hypothesis:

$$
\begin{aligned}
& y_{t}=c+\varepsilon_{t} \\
& \varepsilon_{t}=z_{t} \sqrt{h_{t}} \\
& h_{t}=\alpha_{0}+\alpha_{1} \varepsilon_{t-1}^{2}+\varphi I_{\varepsilon_{t-i<0}} \varepsilon_{t-1}^{2}+\beta_{1} h_{t-1}
\end{aligned}
$$

$$
z_{t} \text { i.i.d.N }(0,1)
$$

Here, the variables $t$ and $z$ are independently, identically distributed (i.i.d) according to a centered and reduced normal distribution. The log-likelihood function of a sample with $T$ observations $\left\{y_{1}, \ldots \ldots, y_{T}\right\}$ obtained under a conditional distribution of $t$ and $y$ knowing its function is written as:

$$
\log L(\rho)=-\frac{T}{2} \log (2 \Pi)-\frac{1}{2} \sum_{t=1}^{T} \log \left(h_{t}(\rho)\right)-\frac{1}{2} \sum_{t=1}^{T} \frac{\left[y_{t}-m_{t}(\rho)\right]^{2}}{h_{t}}
$$

Here, $\rho$ denotes the model's set of parameters, $\rho$ denotes conditional forecast, and $\rho$ denotes conditional variance, respectively.

The case of the GJR-GARCH is presented below:

$$
\begin{aligned}
& m_{t}(\rho)=c \\
& h_{t}(\rho)=\alpha_{0}+\alpha_{1} \varepsilon_{t-1}^{2}+\varphi I_{\varepsilon_{t-i<0}} \varepsilon_{t-1}^{2}+\beta_{1} h_{t-1}
\end{aligned}
$$




\section{Empirical Analysis}

\section{A. Data}

We empirically examine the interaction between stock markets of different increments. The database consists of increments from four countries of Egypt, Malaysia, South Africa, and Turkey. The study makes use of daily returns of market indices during the period of January 1, 1997 to June 15, 2010. The choice of this set of countries is linked to the digital economy ranking.

Figure 1 shows the daily centered logarithmic returns. It is clear that they are highly correlated.

Figure 1. Daily logarithmic centered returns

A. Daily logarithmic centered returns: Egypt

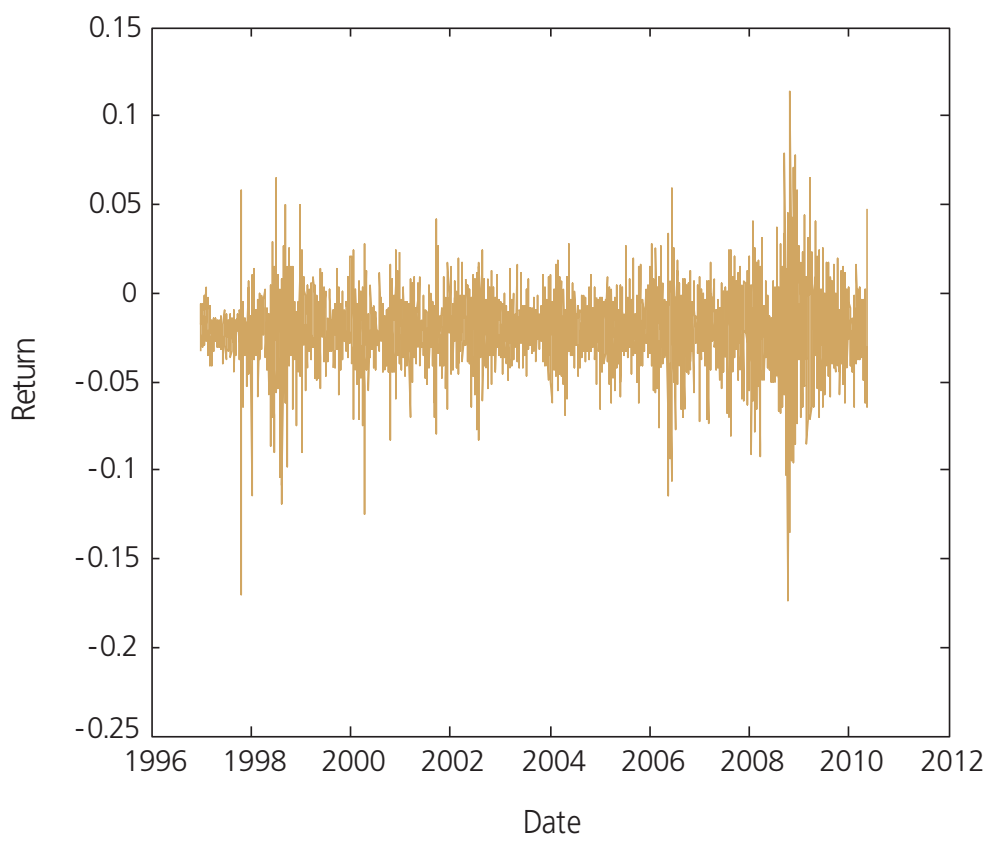



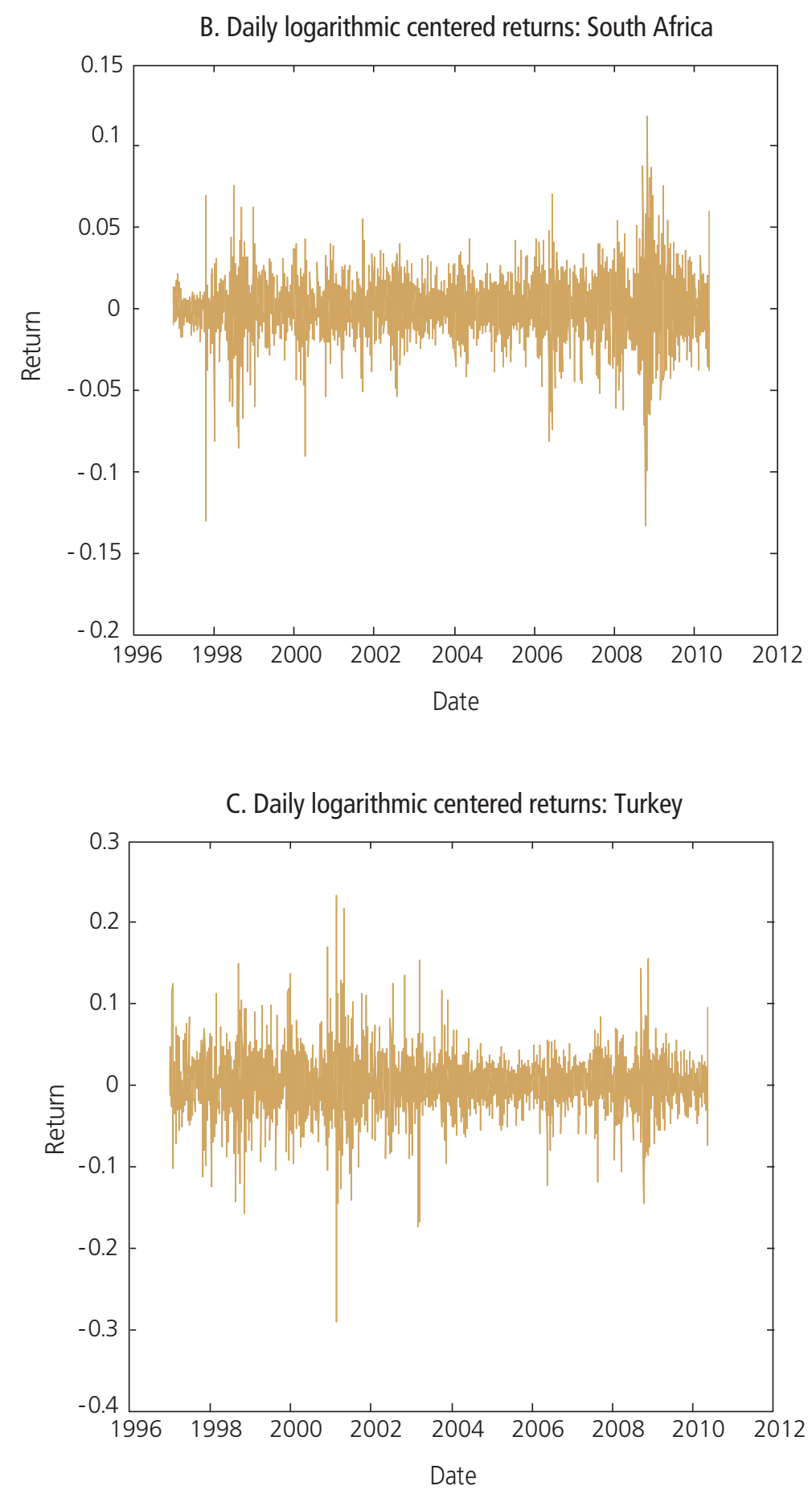


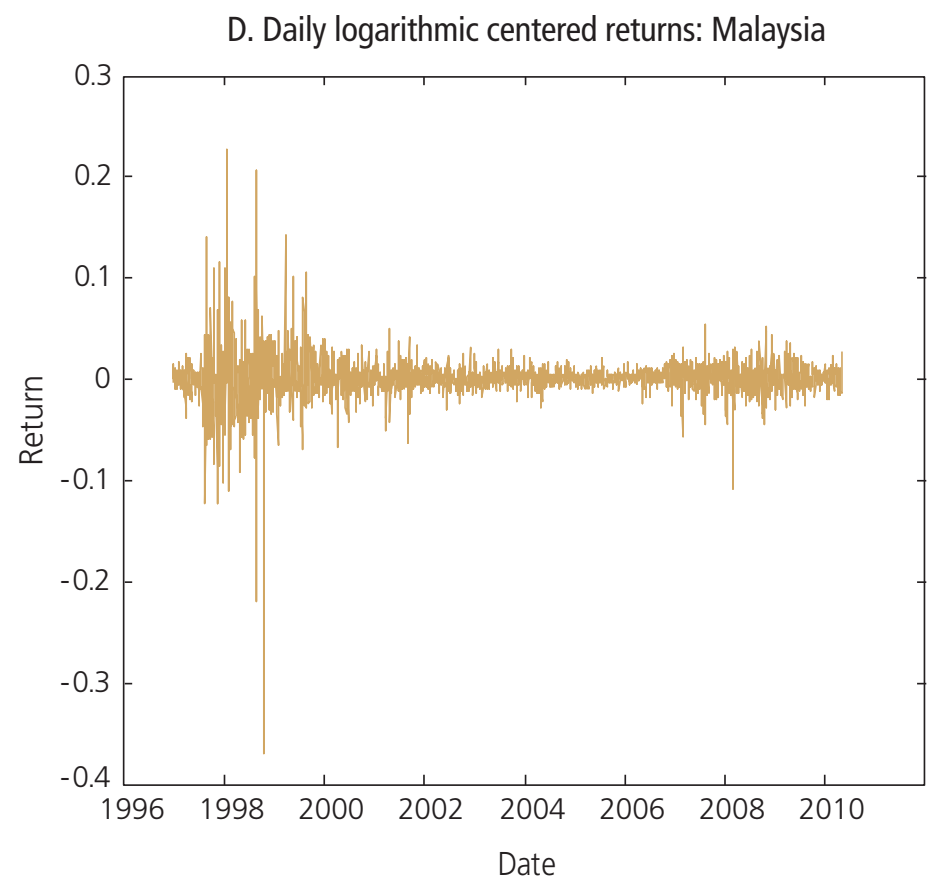

The Extreme Value Theory (EVT) represents a domain of the probability theory that deals with the study of extreme events. Such events are characterized by extreme deviations from the normal median of their probability distributions. More precisely, the EVT studies and models the behavior of distributions in their extreme tails. These rare events are described by a thickening of the tails, which determines an excess of the kurtosis above the characteristic value of the Gaussian distribution. Therefore, the apparitions of the so-called fat tails are also known as the leptokurtic distributions. EVT focuses directly on the tails and, therefore, could potentially give us better estimates and forecasts on risk. Applying EVT to the return series, however, is inappropriate as they are not distributed independently and identically.

Thus, following the approach of Frey and McNeil (2000), we use GARCH model to fit the return series and apply EVT to the residuals rather than to the return series. Figure 2 illustrates the residuals by the conditional standard deviation from each index. We show the zero-mean, while the independent and identically distributed series depend on the EVT estimation. 
Figure 2. Filtered conditional residuals

A. Filtered conditional standard deviations: Egypt

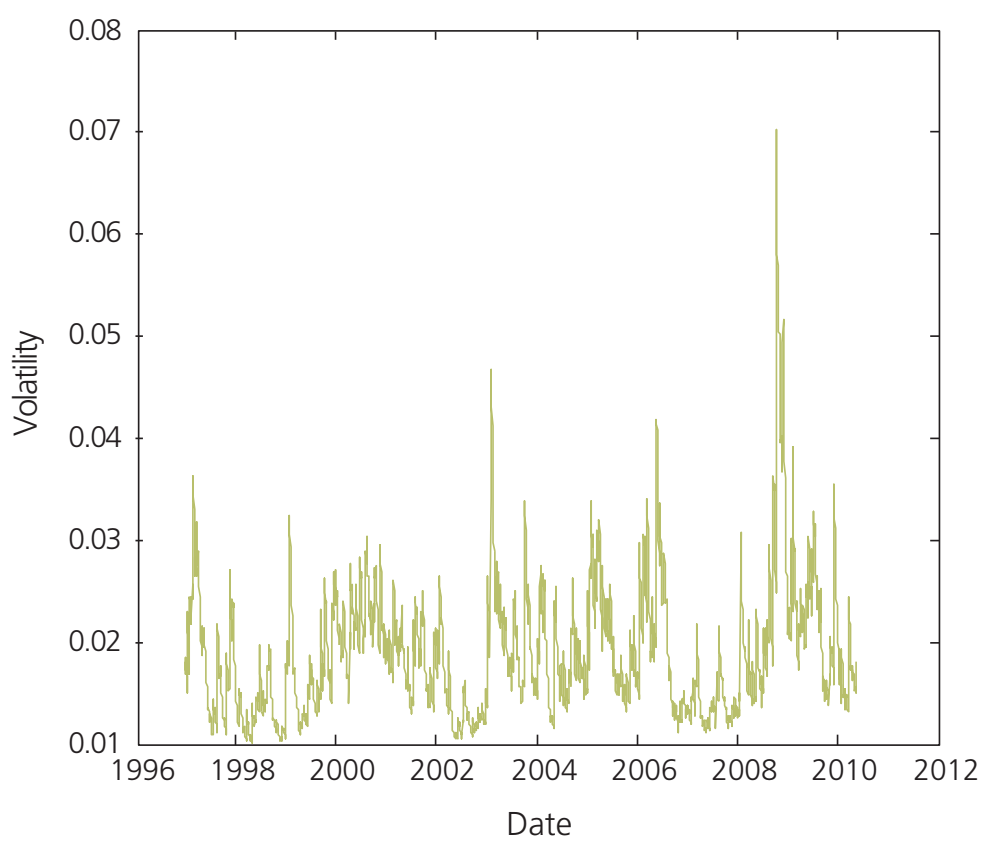

B. Filtered conditional standard deviations: South Africa

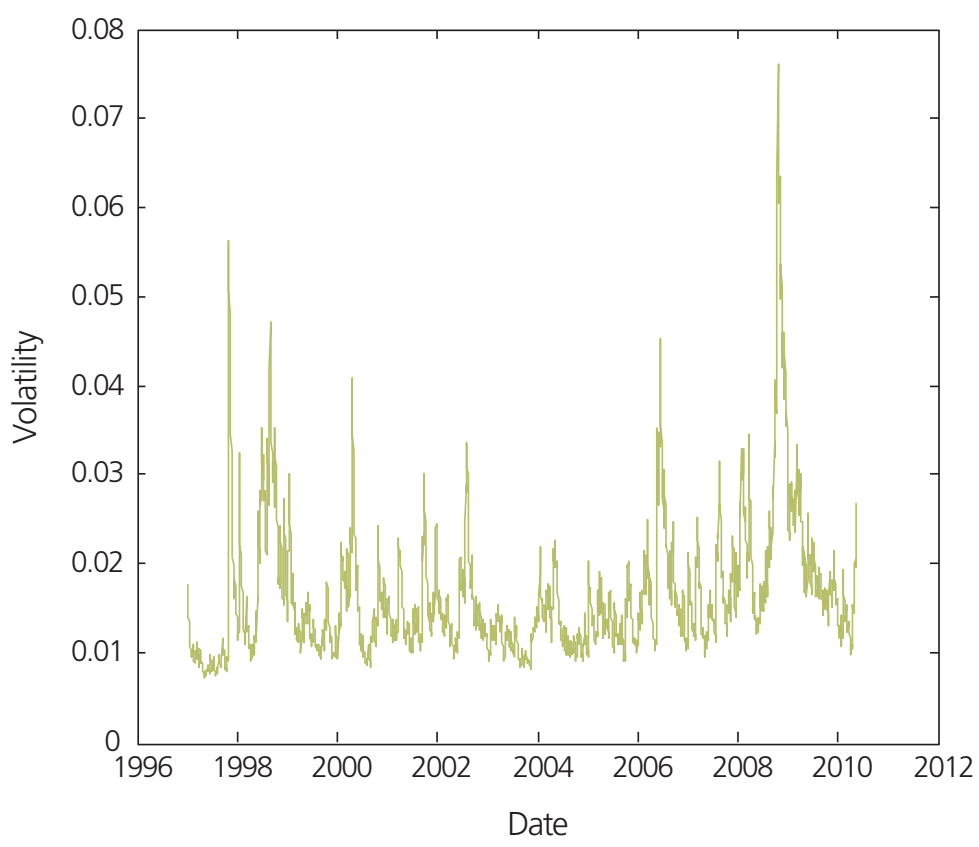




\section{Filtered conditional standard deviations: Turkey}

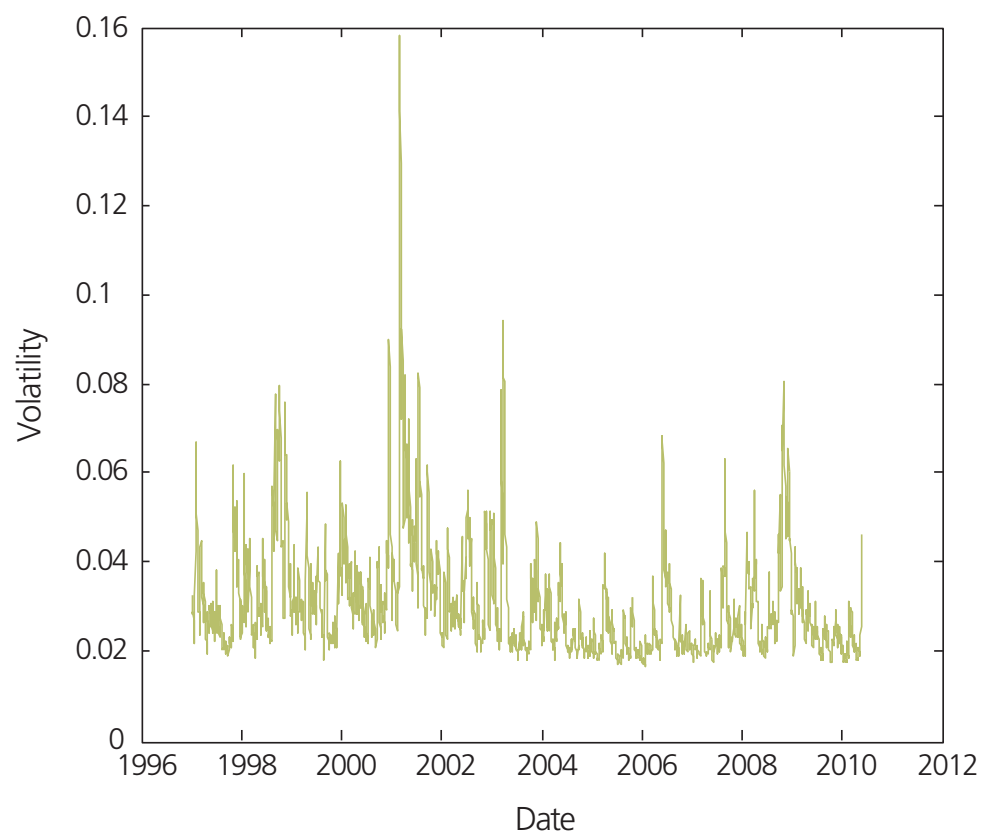

D. Filtered conditional standard deviations: Malaysia

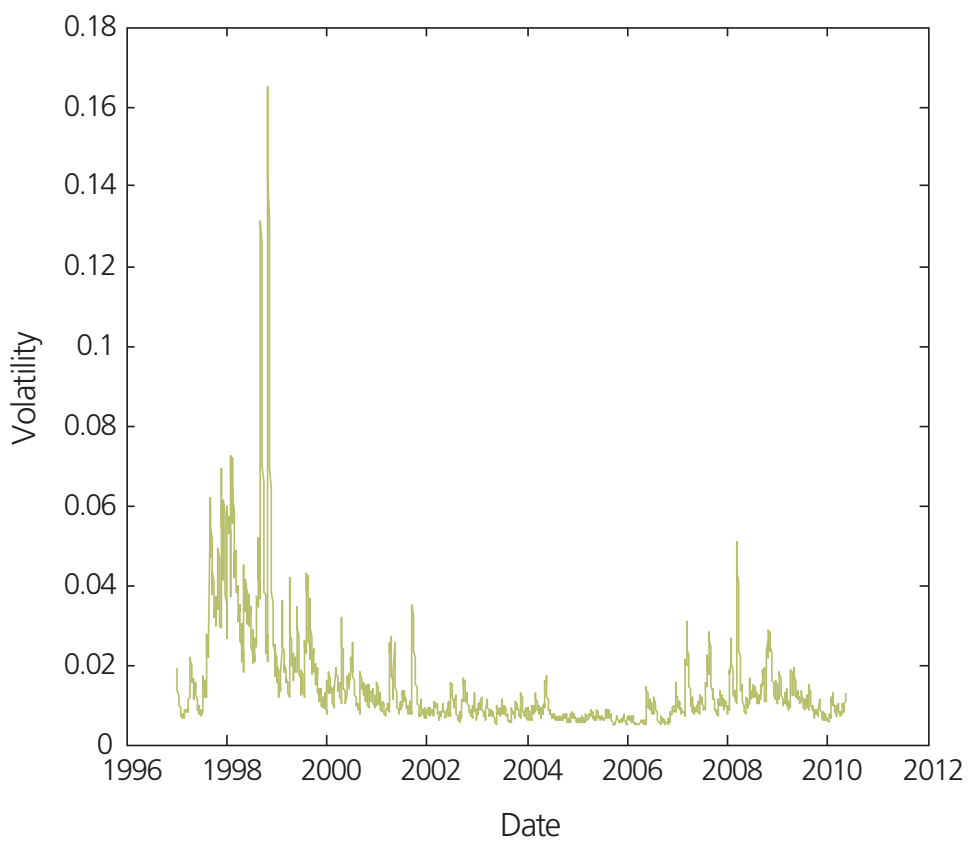




\section{B. Step-wise probability distribution}

We statistically describe probability distribution of daily returns of series of whatever capital increments. We assume that this description is given by a semi-parametric step-wise distribution, where asymptotic behavior in each tail replicates a generalized Pareto distribution. Afterwards, a copula will be used to provide random figures to pilot the simulations. The transformed CDF and the reverse CDF determine the volatility of returns simulated as an element of the diffusion edge of the stochastic differential equation. The average return of each increment is determined by risk less movement and incorporated at the deviation edge of the stochastic differential equation.

Figure 3 presents, respectively, the residuals Autocorrelation Function (ACF) and sample ACF of squared standardized residuals. The residual of ACF indicates periodic smooth correlation. However, the sample ACF of the squared residuals reported in Figure 4 shows an important level of determination, which offer that we have used our GJR-GARCH model to condition data for the tail evaluation process. 


\section{Figure 3. Autocorrelation function of residuals}

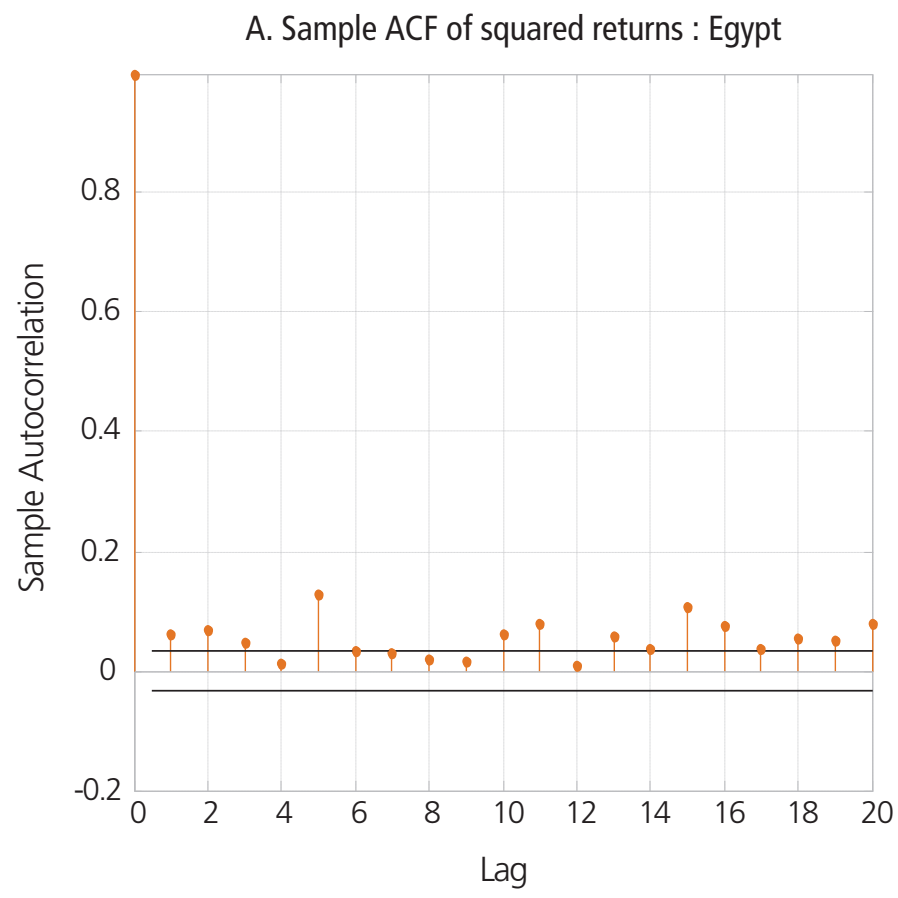

B. Sample ACF of squared returns : South Africa

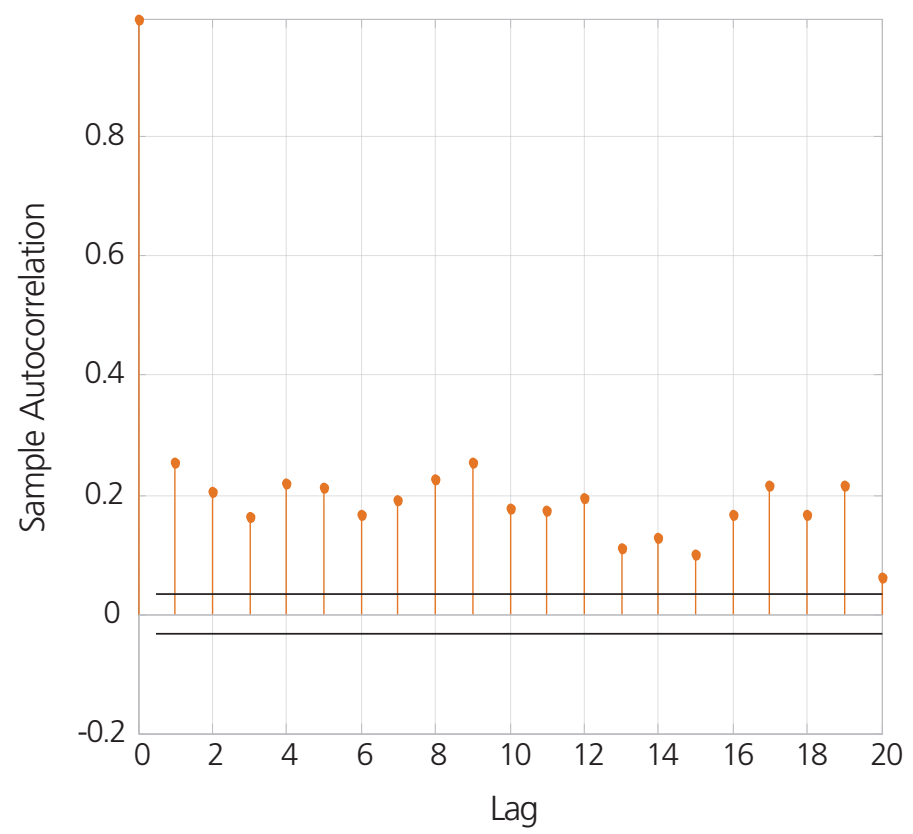



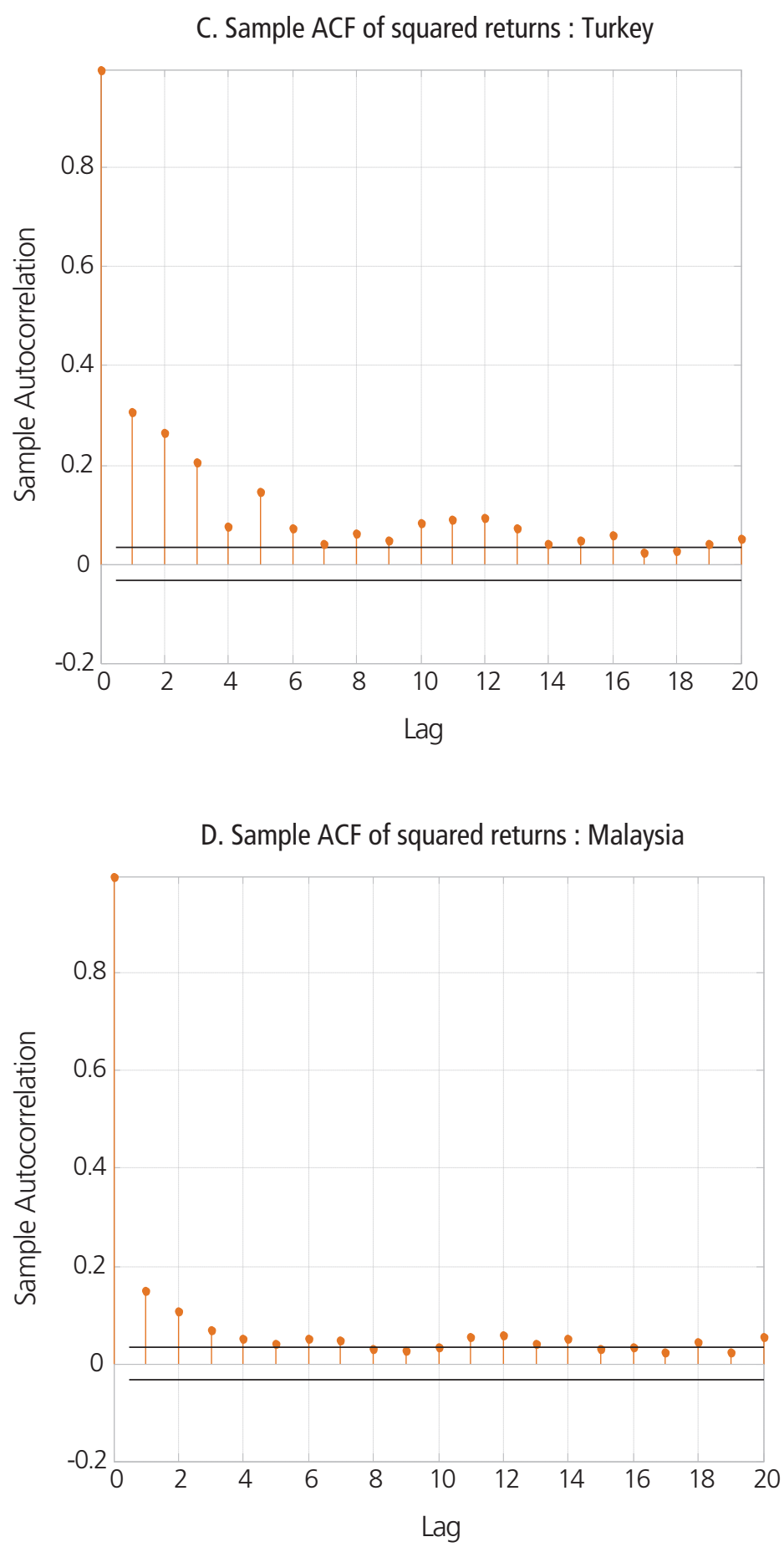


\section{Figure 4. Autocorrelation function of residuals squared}

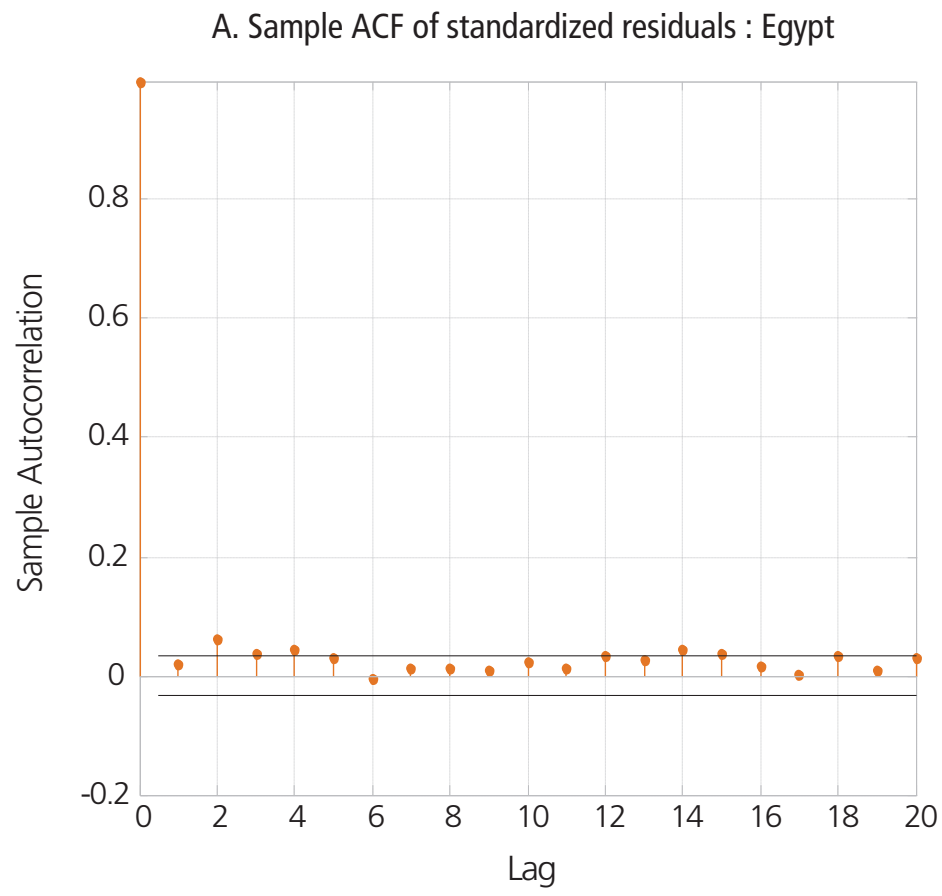

B. Sample ACF of standardized residuals : South Africa

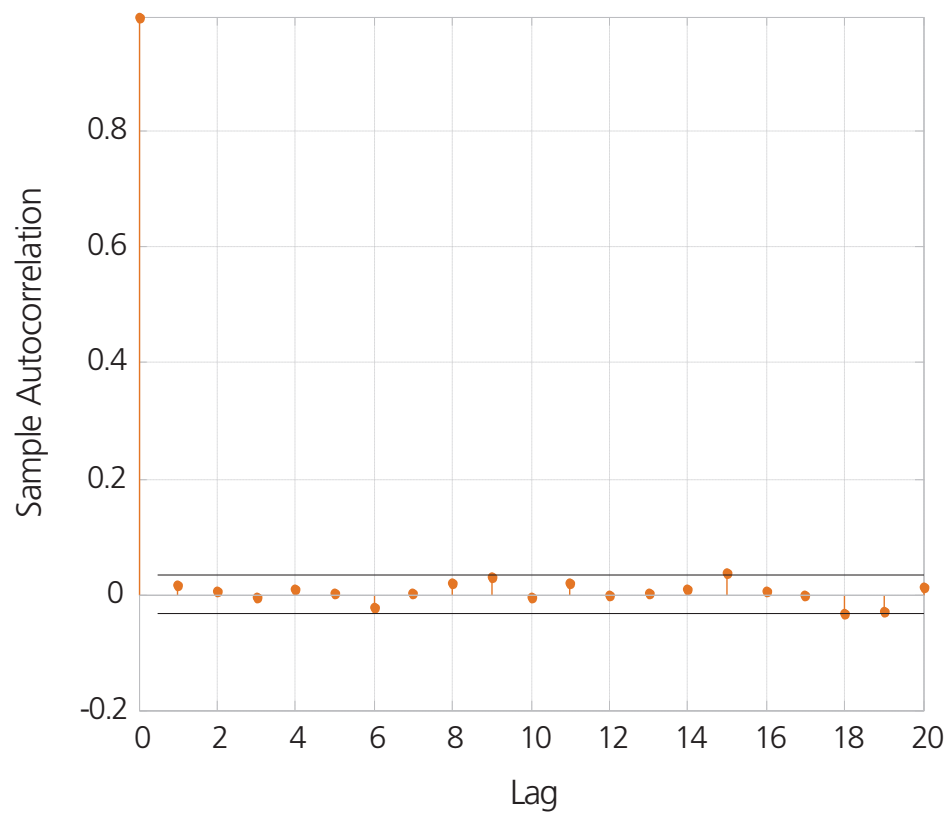



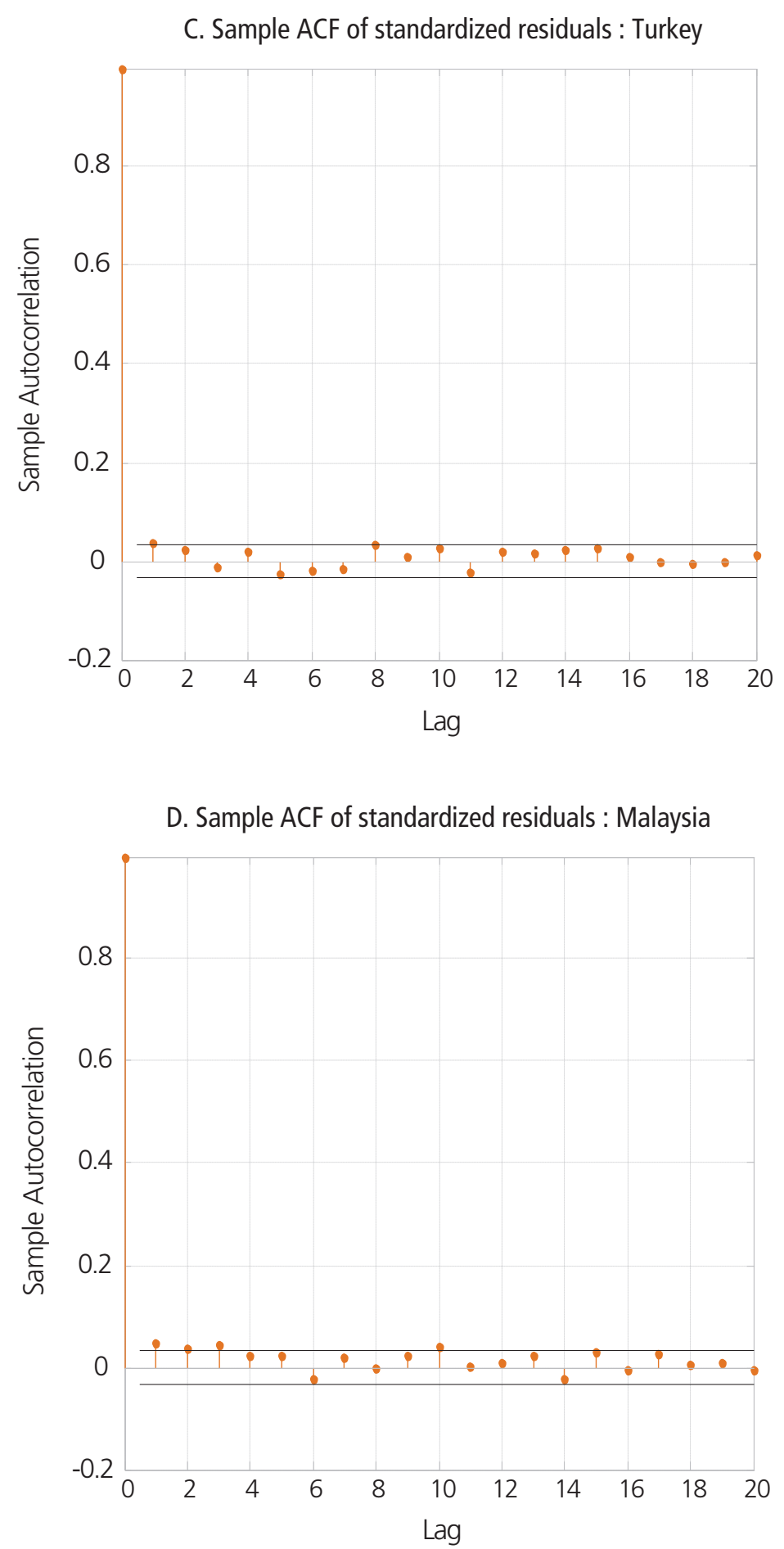


\section{Conditional distribution function estimation}

The Cumulative Distribution Function (CDF), or just distribution function, describes the probability that a real-valued random variable $X$ with a given probability distribution will be found to have a value less than or equal to $x$. In the case of a continuous distribution, it gives the area under the probability density function from minus infinity to $x$. CDFs are also used to specify the distribution of multivariate random variables. Estimating a conditional distribution function is an important feature of many statistical problems, including, for example, the regression analysis of Yin and Cook (2002), where a significant problem is the prediction of a response for a given value of a multivariate explanatory variable. Specific applications include those in economics and finance such as Foresi and Paracchi (1992), Bond and Patel (2000), and Watanabe (2000); in data mining and signal processing such as Adali, Liu, and Sonmez (1997); and a wide range of problems where forecasts are to be made from linear or non-linear time series Fan and Yao (2003)

Given the i.i.d residuals, we present the empirical estimation of CDF. We would clear the estimated CDF by eliminating the biased sample scale CDF. Although the nonparametric estimations of CDF are well-fit for the center of the distribution where most of the data are found, they tend to be inaccurate for the upper and lower tails. To better estimate distribution tails, we apply EVT to these residuals in each tail. Figure 5 presents the semi-parametric CDF of four countries with data other than the one on which modelfit is supported. 
Figure 5. Empirical cumulative distribution function

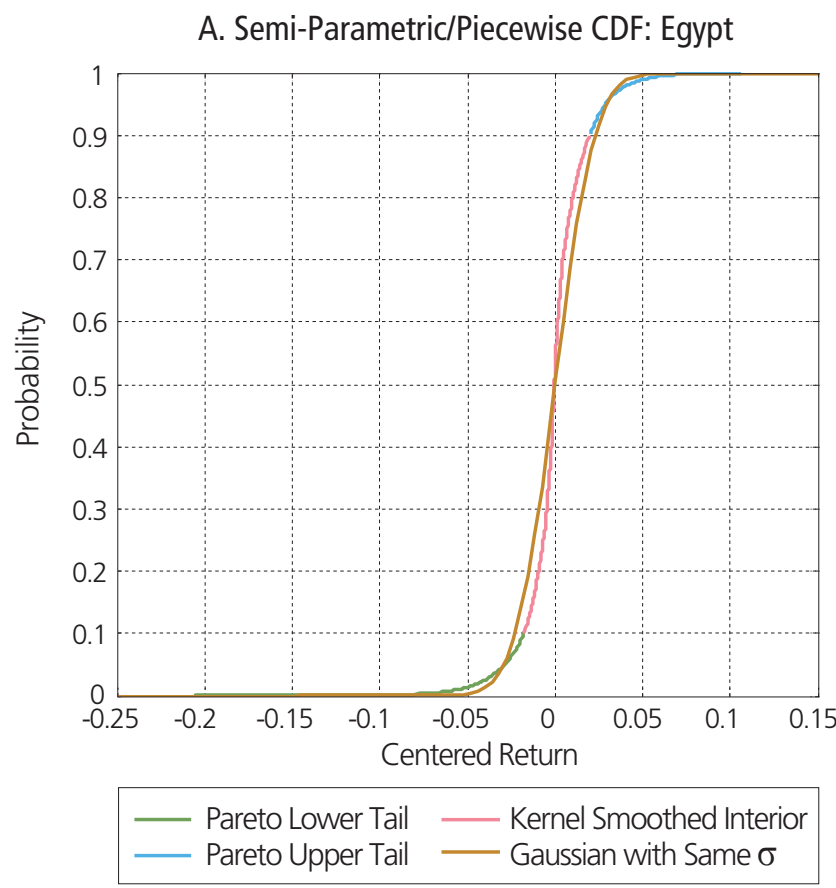

B. Semi-Parametric/Piecewise CDF: South Africa

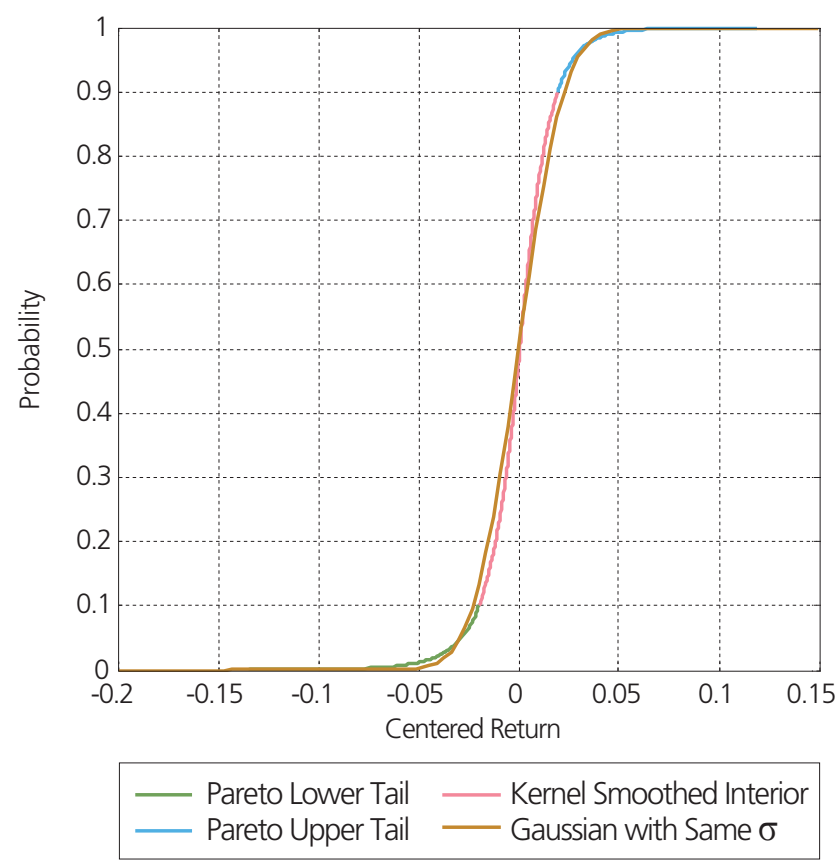



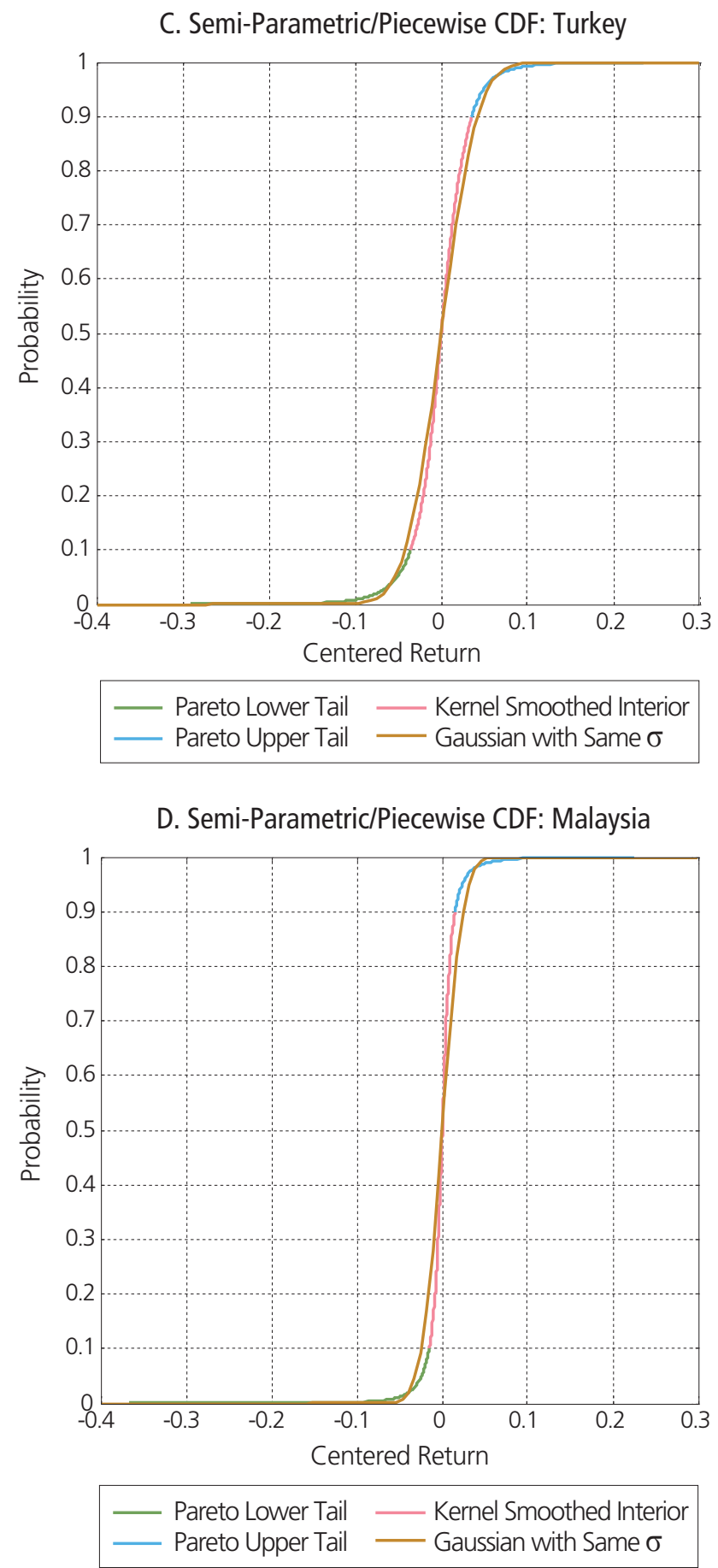
The distribution shows interpolation at the CDF center and extrapolation in each tail. These would allow the estimation of quintiles outside past events and are inestimable for risk management purposes. Our GJR-GARCH model, Gaussian, the Student, and Calyton copulas are used to measure a foreign multi-dimensional exchange portfolio risk. We use the multivariate Gaussian, Student, and Clayton copula to show the correlation structure.

Risk managers are generally more concerned with the probable state of future business as what it would be like next week instead of next year (Hoppe 1998). Consequently, to highlight the importance of recent events, while preserving the econometric validity of data, risk managers may select applying exponential weightings for long chronological series.

We illustrate the diagrams of upper tail estimation residuals. It shows that the GPD adapts the residuals completely.

Figure 6. Upper tail of residuals

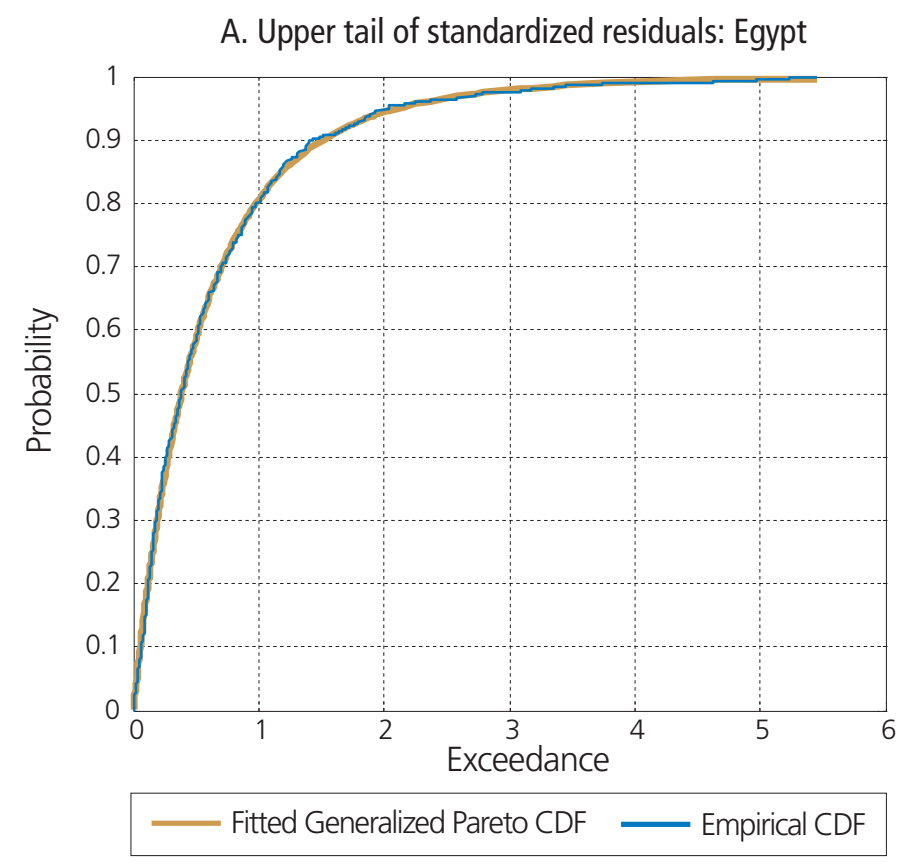




\section{B. Upper tail of standardized residuals: South Africa}

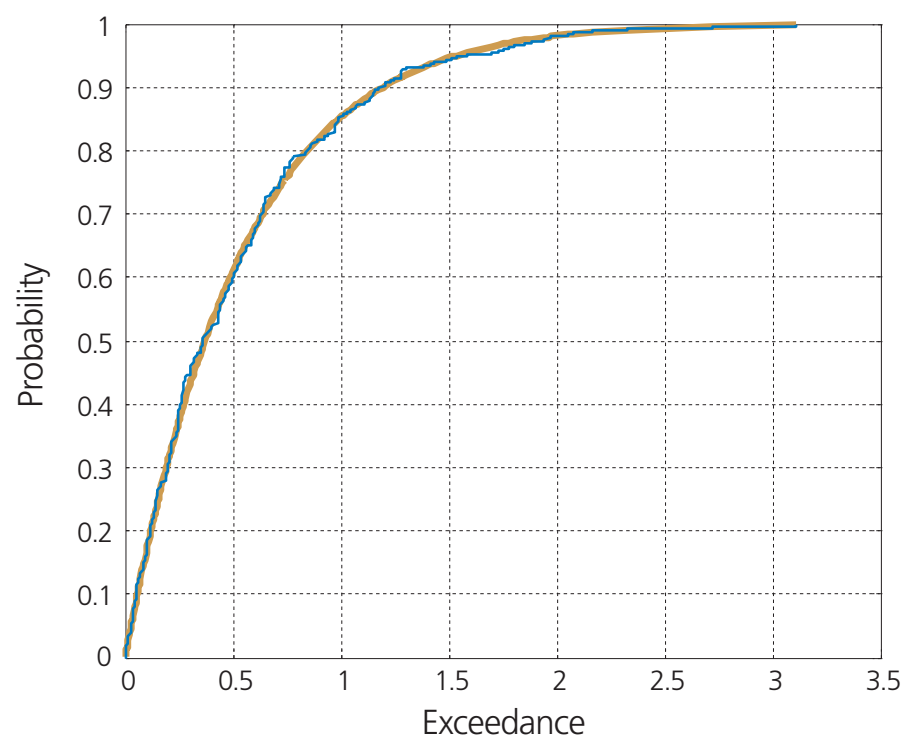

Fitted Generalized Pareto CDF Empirical CDF

\section{Upper tail of standardized residuals: Turkey}

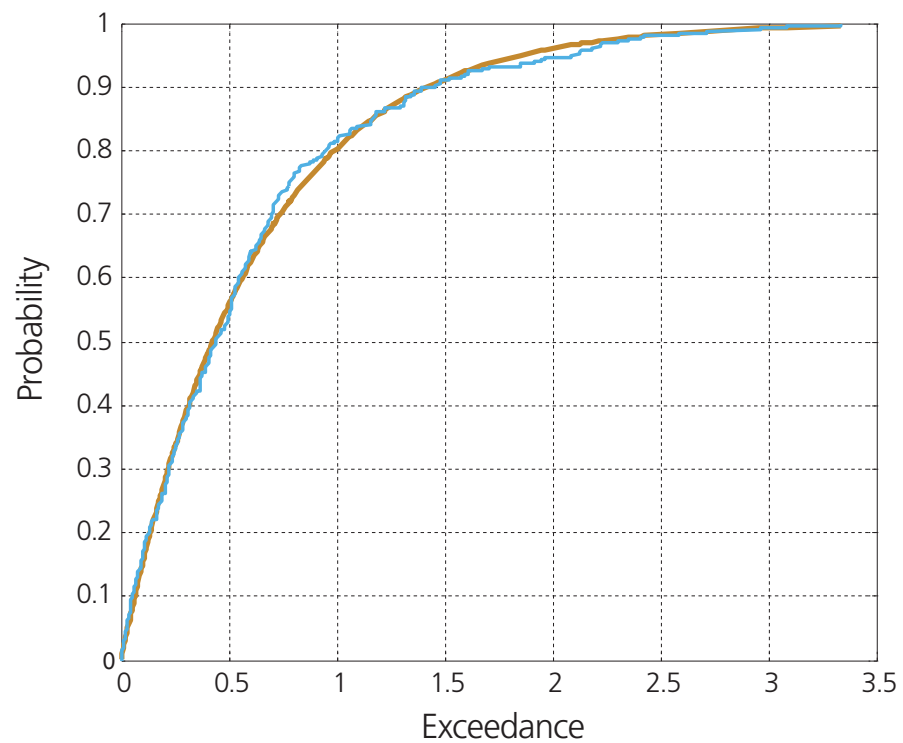

Fitted Generalized Pareto CDF Empirical CDF 


\section{Upper tail of standardized residuals: Malaysia}

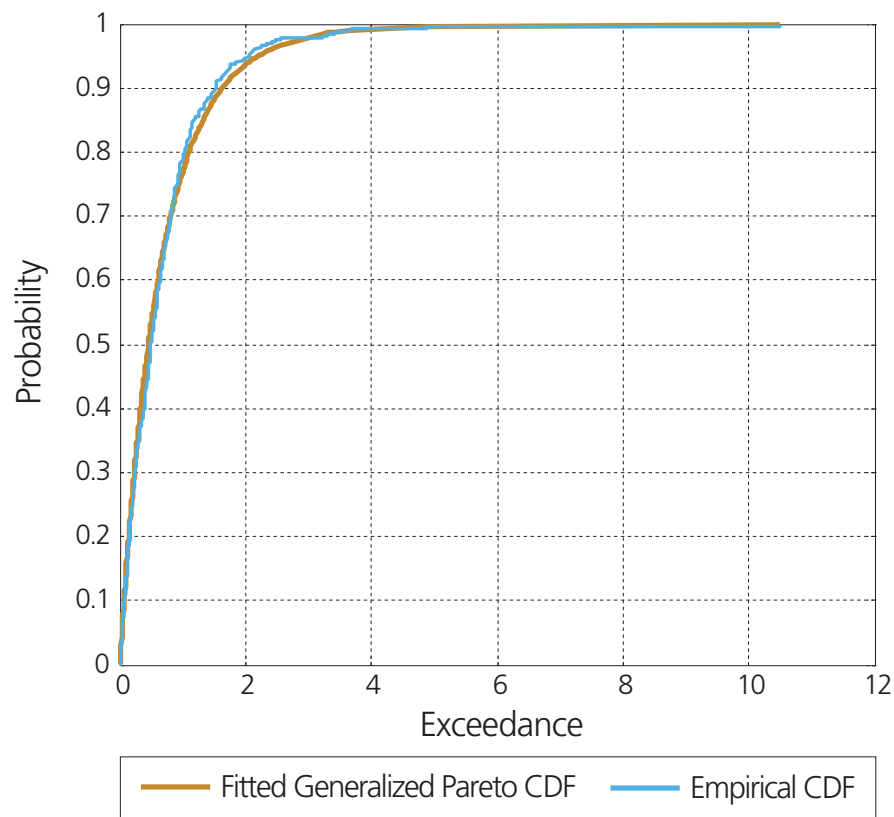

Figure 6 indicate that empirical CDF fits the residuals of exchange rate returns perfectly. In our approach, we extract the filtered residuals from each returns series with a GJRGARCH model, and then we construct the marginal of each series using the empirical CDF for the interior and the GPD estimates for the upper tails. The advantage of this approach is that the i.i.d assumption behind the EVT is less likely to be violated by the filtered series. Fitting the GPD to the filtered returns requires specification of the upper thresholds.

(i) We measure the residuals and the independent returns respectively by using the multivariate copula and the Extreme Value Theory (EVT). (ii) We measure the dependence between variables by the copula and implement the GJR-GARCH used to reintroduce the heteroskedasticity in the returns. (iii) We apply this method to determine the value of VaR. 


\section{Determination of Value at Risk and CVaR}

Next, we standardize the individual residuals of our GJR-GARCH version as varied by the empirical semi-parametric CDF and then adapt the copula to the transformed data. We calculate the empirical $\mathrm{VaR}$ and $\mathrm{CVaR}$ of an equally weighted portfolio. Table 1 shows the VaR and CVaR of the portfolio.

Table 1 indicates that under the same confidence level, VaR and CVaR calculated from Clayton is less than the student and Gaussian Copula. That is because Clayton Copula considers the tail correlation.

Table 1. Portfolio risk under an equally weighted foreign exchange portfolio

\begin{tabular}{|c|c|c|c|c|c|}
\hline & Confidence & $\begin{array}{c}\text { Risk } \\
\text { Value }\end{array}$ & $\begin{array}{c}\text { Gaussian } \\
\text { Copula }\end{array}$ & $\begin{array}{l}\text { Student } \\
\text { Copula }\end{array}$ & $\begin{array}{l}\text { Clayton } \\
\text { Copula }\end{array}$ \\
\hline \multirow{6}{*}{$\begin{array}{l}\text { Proposed } \\
\text { Approach }\end{array}$} & \multirow{2}{*}{0.90} & VaR & 0.0031 & 0.0029 & 0.0028 \\
\hline & & $\mathrm{CVaR}$ & 0.0045 & 0.0032 & 0.0031 \\
\hline & \multirow{2}{*}{0.95} & VaR & 0.0043 & 0.0035 & 0.0033 \\
\hline & & CVaR & 0.0060 & 0.0049 & 0.0041 \\
\hline & \multirow{2}{*}{0.99} & VaR & 0.0059 & 0.0050 & 0.0044 \\
\hline & & CVaR & 0.0079 & 0.0068 & 0.0064 \\
\hline \multirow{6}{*}{$\begin{array}{c}\text { Xubiao } \\
\text { Approach }\end{array}$} & \multirow{2}{*}{0.90} & $\mathrm{VaR}$ & 0.0029 & 0.0024 & 0.0024 \\
\hline & & $\mathrm{CVaR}$ & 0.0043 & 0.0037 & 0.0035 \\
\hline & \multirow{2}{*}{0.95} & VaR & 0.0038 & 0.0032 & 0.0031 \\
\hline & & CVaR & 0.0053 & 0.0046 & 0.0042 \\
\hline & \multirow{2}{*}{0.99} & $\mathrm{VaR}$ & 0.0061 & 0.0058 & 0.0049 \\
\hline & & CVaR & 0.0081 & 0.0070 & 0.0060 \\
\hline
\end{tabular}

(Source) Xubiao (2009).

\section{Conclusion}

In this paper, we apply the GJR-GARCH with the Extreme Value Theory (EVT) 
to design the returns distribution model. (i) Our approach uses the GJR-GARCH to estimate conditional average of each asset. (ii) Our method is adjusted to residuals tail distribution. (iii) Our structure may also be applied to other Value at Risk (VaR) and Conditional Value at Risk ( $\mathrm{CVaR})$ portfolio problems. The results of this study could be used to execute a good risk management of global investments.

Future work may examine a combination of different copulas in dependence structure.

Received 16 May 2013 , Revised 10 February 2014, Accepted 22 December 2014

\section{References}

Aboura, Sofoane and Wagner Niklas. "Systematic: Credit Risk: CDX Index Correlation and Extreme Dependence." in Credit Risk: Models, Derivatives, and Management, ed. by N. Wagner, Chapman and Hall/CRC, Boca Raton (2008): 377-390.

Adali, Tulay, Liu, Xiao and Sonmez, Kemal. "Conditional distribution learning with neural networks and its application to channel equalization." IEEE Trans. Signal Processing (1997):1051-1064.

Ane, Thierry, and Kharoubi, Cécile. "Dependence Structure and Risk Measure.” Journal of Business, (2003): 411-438.

Ang Andrew and Chen Joseph. "Asymmetric correlations of equity portfolios." Journal of Financial Economics, 63, no.3 (2002): 443-494.

Bollerslev, Tim Peter. " Generalized Autoregressive Conditional Heteroskedasticity." Journal of GARCH, (1986): 307-327.

Bond, Shaun A and Patel, Kanak. "The conditional distribution of real estate returns: Relating time variation in higher moments to downside risk measurement." Technical report, Dept. Land Economy, Univ. Cambridge, 2000.

Bouyè, Eric, Durrleman, Vado, Nikeghbali, Ashan, Riboulet, Gael and Roncalli, Thierry. "Copulas for finance, a reading guide and some applications." Working paper, Financial Econometrics Research Center, City University, London, 2000. 
Breymann, Wolfgang, Dias, Alexandra and Embrechts, Paul. "Dependence structures for multivariate high-frequency data in Finance." Quantitative Finance 3, no.1 (2003): $1-14$.

Chen, Xiaohong, Fan, Yanqin and Patton, Andrew. "Simple tests for models of dependence between multiple financial time series, with applications to U.S. equity returns and exchange rates," Working Paper, New York University, Vanderbilt University and London School of Economics, 2004.

Cherubini, Uberto and Luciano, Elisa. "Value-at-risk trade-off and capital allocation with copulas." Economic Notes 30, no.2, (2001): 235-256.

Das, Sanjiv., Duffie Darrel., Kapadia Nikunj and Saita. Lendro. "Common Failings: How Corporate Defaults are Correlated." Journal of Finance 62,no.1 (2007): 93-117.

Dembo, Ron and Andrew Freeman. "The Rules of Risk." John Wiley and Sons. p. 10. ISBN 0-471-40163-3, 2001.

Dowd, Kevin, Beyond. "Value-at-Risk: The New Science of Risk Management," John Wiley and Sons, London, 1998.

Embrechts, Paul, McNeil, Alexander and Straumann, Daniel. "Correlation and dependence in risk management: properties and pitfalls.” In: Risk Management: Value at Risk Beyond. ed. M.A.H. Dempster, Cambridge University Press, Cambridge (2002): 176-223.

Embrechts, Paul and Hoing, Andrea, Juri, Alessandro. "Using copula to bound the valueat-risk for functions of dependent risks." Finance and Stochastics (2003): 145-167.

Embrechts, Paul, McNeil, Alexander and Frey Rudiger. "Quantitative Risk Management." University Press Princeton, 2005.

Engle Robert. "Autoregressive Conditional Heteroskedasticity with Estimates of the Variance of United Kingdom Inflation.” Econometrica (1982): 987-1007.

Foresi, and Paracchi. "The conditional distribution of excess returns: An empirical analysis.” Working Paper C. V. Starr Center, New York Univ (1992): 92-49.

Fortin, Ines and Kuzmics, Christoph. "Tail dependece in stock return pairs." International Journal of Intelligent Systems in Accounting, Finance and Management (2002); 89-107. 
Georges, Pierre, Lamy, Arnaud Guilhem, Nicolas, Emeric, Quibel, Guillaume and Roncalli, Thierry. "Multivariate survival modelling: a unified approach with copulas." Working paper, Credit Lyonnais, Paris, 2001.

Glosten, Lawrence, Jagannathan, Ravi. and Runkle, David. "On the Relation between the Expected Value and the Volatility of the Nominal Excess Return on Stocks." Journal of Finance, American Finance Association, 48, no 5. (1993): 1779-1801.

Hoppe, Richard. "VaR and the unreal world." Risk, 11, no.7, (1998): 45-50.

Hougaard, Philip. "A class of multivariate failure time distributions." Biometrika, (1986): 671-678.

Huang, Jen-Jsung, Lee, Kuo-Jung, Hueimei, Liang. and Lin, Wie-Fu, "Estimating value at risk of portfolio by conditional copula-GARCH method," Insurance: Mathematics and Economics, 45,no.3, (2009): 315-324.

Joe, Harry Multivariate Models and Dependence Concepts. Chapman and Hall, 1997.

Jondeau, Eric and Rockinger, Michael. "Conditional dependency of 5nancial series: an application of copulas."

Working paper, Banque de France, NER\#82, 2001.

Jondeau, Eric and Rockinger, Micheal. "The copula-GARCH model of conditional dependencies: An international stock market application." Journal of International Money and Finance, 25, no.5 (2006): 827-853.

Jorion, Philippe. "Risk: Measuring the Risk in Value at Risk." Financial Analysts Journal, 52, no.6 (1996): 47-56.

Juri, Alessandro and Wuthrichts, Mario. "Copula convergence theorems for tail events." Insurance: Mathematics and Economics, 30, no.3 (2002): 405-420.

Longin, François and Solnik. Bruno. "Extreme Correlation of International Equity Markets.” Journal of Finance, 56, no.2 (2001): 649-676.

Lindskog, Fillip, and Alexander McNeil. "Common Poisson shock models: applications to insurance and credit risk modelling." ETH preprint. 2001.

Malevergne, Yannick, and Sornette. "Didier Testing the Gaussian copula hypothesis for financial as-sets dependences." Quantitative Finance, (2003): 231-250. 
McNeil Alexander, Rüdiger Frey. "Estimation of tail-related risk measures for heteroscedastic financial time series: an extreme value approach." Journal of Empirical Finance, (2002):271-300.

Meneguzzo, David and Vecchiato, Walter. "Copulas sensitivity in collaterized debt obligations and basket defaults swaps pricing and risk monitoring." Working paper, Veneto Banca, 2003.

Mendes, Beatriz Vaz Melo, and Souza, Rafael Martins. "Measuring Financial Risks with Copulas." International Review of Financial Analysis, 13, no.1 (2004): 27-45.

Nelson, Daniel. "Conditional heteroskedasticity in asset returns: a new approach." Econometrica, 59,no.2 (1991): 347-370.

Nelsen, Roger. "Introduction to Copulas.” Springer Verlag, 1999.

Palaro Helder, and Hotta, Luiz Koodi. "Using Conditional Copulas to Estimate Value at Risk.” Journal of Data Science, 4, no.1 (2006): 93-115.

Patton, Andrew. "Modelling asymmetric exchange rate dependence." Working paper, University of California, San Diego, 2003a.

Patton, Andrew. "Estimation of multivariate models for time series of possibly different lengths," Working paper, University of California at San Diego, 2003.

Patton, Andrew Modelling "asymmetric exchange rate dependence." International Economic Review 74, no.2 (2006): 527-556.

Rodriguez, Juan Carlos."Measuring financial contagion: A Copula Approach.” Journal of Empirical Finance 14, no.3 (2007):401-423.

Trivedi, Pravin, Zimmer David. "Copula Modeling: An introduction for Practitioners. Foundations and Trends in Econometrics.” 1, no.1 (2007): 1-111.

Van den Goorbergh, Rob. "A copula-based autoregressive conditional dependence model of international stock markets.” DNB Working Paper No. 22, 2004.

Wang, Zong.Run, Chen, and Zhou, Yan. Ju. "The exchange rate risk of Chinese Yuan: using VaR and ES based on extreme value theory." Journal of Applied Statistics 37/2, (2010): 265-282.

Watanabe, Toshiaki. "Excess kurtosis of conditional distribution for daily stock returns: 
The case of Japan." Applied Economics Letters. (2000): 353-355.

Xubiao, He, and Gong, Pu. "Measuring the coupled risks: A copula-based CVaR model." Journal of Computational and Applied Mathematics, (2009): 1066-1080.

Yin, Xiangrong and Cook, "Dennis. Dimension reduction for the conditional kth moment in regression.” J. R. Stat. Soc. Ser. B Stat. Methode (2002): 159-175. 